\title{
The rectal mucosa and condomless receptive anal intercourse in HIV-negative MSM: implications for HIV transmission and prevention
}

\author{
CF Kelley ${ }^{1,2}$, CS Kraft ${ }^{1,3}$, TJB de Man $^{4}$, C Duphare ${ }^{5}$, H-W Lee ${ }^{5}$, J Yang ${ }^{6}$, KA Easley ${ }^{6}$, GK Tharp ${ }^{5}$, \\ MJ Mulligan', PS Sullivan ${ }^{2}$, SE Bosinger ${ }^{5}$ and RR Amara ${ }^{5,7}$
}

Most HIV transmissions among men who have sex with men (MSM), the group that accounted for $67 \%$ of new US infections in 2014, occur via exposure to the rectal mucosa. However, it is unclear how the act of condomless receptive anal intercourse (CRAI) may alter the mucosal immune environment in HIV-negative MSM. Here, we performed a comprehensive characterization of the rectal mucosal immune environment for the phenotype and production of proinflammatory cytokines by CD4 and CD8 T cells, global transcriptomic analyses, and the composition of microbiota in HIV-negative MSM. Our results show that compared with men who had never engaged in anal intercourse, the rectal mucosa of MSM engaging in CRAI has a distinct phenotype characterized by higher levels of Th17 cells, greater CD8 + T cell proliferation and production of pro-inflammatory cytokines, molecular signatures associated with mucosal injury and repair likely mediated by innate immune cells, and a microbiota enriched for the Prevotellaceae family. These data provide a high-resolution model of the immunological, molecular, and microbiological perturbations induced by CRAI, will have direct utility in understanding rectal HIV transmission among MSM, and will enhance the design of future biomedical prevention interventions, including candidate HIV vaccines.

\section{INTRODUCTION}

Men who have sex with men (MSM) continue to make up the largest risk group for HIV infection in the US, accounting for $67 \%$ of new infections in $2014,{ }^{1}$ underscoring the urgent need for effective HIV prevention interventions for this population. Although pre-exposure prophylaxis with daily or on-demand oral tenofovir/emtricitabine is highly effective in preventing HIV infection among MSM, ${ }^{2}$ additional prevention options including an effective vaccine will be necessary to end HIV transmission. One potential barrier to the effectiveness of biomedical HIV prevention interventions is the relative ease of HIV transmission across the rectal mucosa, where $~ 70 \%$ of infections are thought to occur among MSM. ${ }^{3,4}$

Previous studies have examined the effects of coitus on the female genital tract ${ }^{5}$; however, several important differences indicate that rectal mucosa is more susceptible to HIV acquisition: (i) the single-layer columnar mucosal epithelium of the rectum is likely more prone to mechanical trauma during sexual intercourse than the vagina or penis, ${ }^{4,6}$ (ii) the gastrointestinal tract houses the majority of the body's lymphocyte population, ${ }^{7}$ and (iii) many of these cells are primary target cells for HIV infection (i.e., CD4 + CCR5 + T cells), ${ }^{8}$ and (iv) the most distal section of the rectum harbors substantially greater concentrations of CCR5 expressing macrophages compared with more proximal colonic regions and may be particularly vulnerable to HIV infection. ${ }^{9}$ Overall, current models estimate that these factors contribute to the $\sim 18$-fold increase in HIV transmission probability per exposure event for rectal vs. vaginal exposure and the approximate 50 -fold increase for rectal vs. penile exposure. ${ }^{10}$

${ }^{1}$ Division of Infectious Diseases, Department of Medicine, Emory University School of Medicine, Atlanta, Georgia, USA. ${ }^{2}$ Department of Epidemiology, Rollins School of Public Health, Emory University, Atlanta, Georgia, USA. ${ }^{3}$ Department of Pathology, Emory University School of Medicine, Atlanta, Georgia, USA. ${ }^{4}$ Division of Healthcare Quality Promotion, Centers for Disease Control and Prevention, Atlanta, Georgia, USA. ${ }^{5}$ Division of Microbiology and Immunology, Yerkes National Primate Research Center, Emory University, Atlanta, Georgia, USA. ${ }^{6}$ Department of Biostatistics and Bioinformatics, Rollins School of Public Health, Emory University, Atlanta, Georgia, USA and ${ }^{7}$ Department of Microbiology and Immunology, Emory University School of Medicine, Atlanta, Georgia, USA. Correspondence: CF Kelley (colleen.kelley@emory.edu). 
As the response of the rectal mucosa to condomless receptive anal intercourse (CRAI) in MSM is unlikely to mirror the female genital tract given the differences in the structure of the mucosal barrier, sex differences in the hormonal context, and differences in the microbiota, and since no prior studies have examined the specific effects of CRAI on the rectal mucosa, further examination of the effect of CRAI on the rectal mucosa particularly among MSM is warranted to inform biomedical HIV prevention efforts. Therefore, the goal of this study was to characterize the rectal mucosal immune environment and microbiota among MSM engaging in CRAI as compared with men who had never engaged in anal intercourse.

\section{RESULTS}

\section{The clinical cohort}

Forty-one HIV-negative MSM engaging in CRAI with an HIV-negative partner and 21 men who never engaged in anal intercourse (controls) were enrolled into a study with peripheral blood and rectal biopsy sampling timed with acts of CRAI for MSM engaging in CRAI. For the first biopsy visit, MSM engaging in CRAI were asked to abstain from CRAI for at least $72 \mathrm{~h}$ before the visit. For the second study visit, MSM engaging in CRAI were asked to engage in CRAI within $24 \mathrm{~h}$ of the visit. Demographic and clinical characteristics are presented in Table 1. During study visit 1, one MSM engaging in CRAI was diagnosed with rectal Chlamydia and one was positive for herpes simplex virus type 2 shedding. During study visit 2 , one MSM engaging in CRAI was diagnosed with rectal Chlamydia and one with rectal Gonorrhea.

Rectal CD8 + T cells from MSM engaging in CRAl show greater proliferation status in vivo compared with men that do not engage in Al

To understand the influence of CRAI on the phenotype of memory CD4 and CD8 T cells in the rectum and blood, we measured the expression of Ki67 (indicator of proliferation status), CD38 (indicator of activation status), CCR5 (co-receptor for HIV), and $\alpha 4 \beta 7$ (gut homing potential and facilitates HIV infection ${ }^{11}$; Figure 1 and Table 2). We chose these markers to determine the frequency of activated $\mathrm{T}$ cells and potential HIV target cells. Representative gating strategy for gut is presented in Figure $\mathbf{1}$ and for blood in Supplementary Figure 1 online. Analyses of rectal mucosal mononuclear cells showed a lower percentage of CD $4+\mathrm{CD} 38+$ cells in MSM engaging in CRAI compared with controls (39.9 vs. 50.6\%, $P=0.03$ ); and no differences for CD4 + T cells between MSM engaging in CRAI and controls for expression of CCR5, Ki67, and $\alpha 4 \beta 7$, or co-expression of CCR5 and Ki67. There was a significant decline in the percentage of memory CD4+ $\mathrm{CD} 38+\mathrm{T}$ cells and CD8 + CD38 $+\mathrm{T}$ cells in MSM engaging in CRAI between visit 1 and 2 (adjusted model-based mean for memory CD $4+\mathrm{CD} 38+45.3$ vs. $34.4 \% ; P=0.005$; for memory $\mathrm{CD} 8+\mathrm{CD} 38+71.0$ vs. $63.7 \% ; P=0.01)$. Overall, these data show there is no clear increase in HIV target cell availability in the rectal mucosa of MSM engaging in CRAI compared with controls. However, memory CD8 $+\mathrm{T}$ cells from MSM engaging in CRAI did show higher expression of Ki67 as compared with controls (model-based mean 4.1 vs. 2.6\%; $P=0.03$ ), but no difference between visit 1 and 2 for MSM engaging in CRAI.

There were no significant differences in the percentage of blood CD8 + T cells that expressed Ki67, demonstrating that the higher CD8 + T-cell proliferation in MSM engaging in CRAI is restricted to the rectum (see Supplementary Figure 1 and Supplementary Table 1). Among MSM, enema use was associated with higher expression of CD38 on CD4 + T cells (model-based mean 45.8 vs. 35.1; $P=0.03$ ), but no other cellular phenotypes examined. Frequency of sex was not associated with cellular phenotypes examined. Between visit 1 and 2 , the percentage of CD $4+\alpha 4 \beta 7^{\text {high }}$ cells in the blood increased a small, but statistically significant amount for MSM engaging in CRAI (model-based mean 8.5 vs. $10.8 \%$; $P=0.005)$, and the percentage of CD $4+\mathrm{CD} 38+$ cells decreased (model-based mean 21.2 vs. $17.3 \%$; $P=0.02$ ) for MSM engaging in CRAI.

Table 1 Demographic and clinical characteristics of 41 HIV-negative MSM engaging in CRAl and 21 men who never engaged in Al (controls) included in the study

\begin{tabular}{|c|c|c|c|}
\hline Characteristic & MSM engaging in CRAI $(n=41)$ & Men never engaged in $\mathrm{Al}(n=21)$ & $P$ value \\
\hline Median age in years (25th, 75th) & $28(25.5,33.9)$ & $24(23.5,30.0)$ & 0.02 \\
\hline \multicolumn{4}{|l|}{ Race n (\%) } \\
\hline White & $33(80.0)$ & $14(66.7)$ & - \\
\hline Black & $6(14.6)$ & $2(9.5)$ & - \\
\hline Other & $2(4.9)$ & $5(23.8)$ & 0.11 \\
\hline Lubricant use $n(\%)$ & $39(95.1)$ & NA & - \\
\hline Enema use $n(\%)$ & $18(43.9)$ & NA & - \\
\hline Median CRAI episodes in previous month (25th, 75th) & $5(5,8)$ & NA & - \\
\hline
\end{tabular}

Abbreviations: Al, anal intercourse; CRAl, condomless receptive anal intercourse; MSM, men who have sex with men. 
a
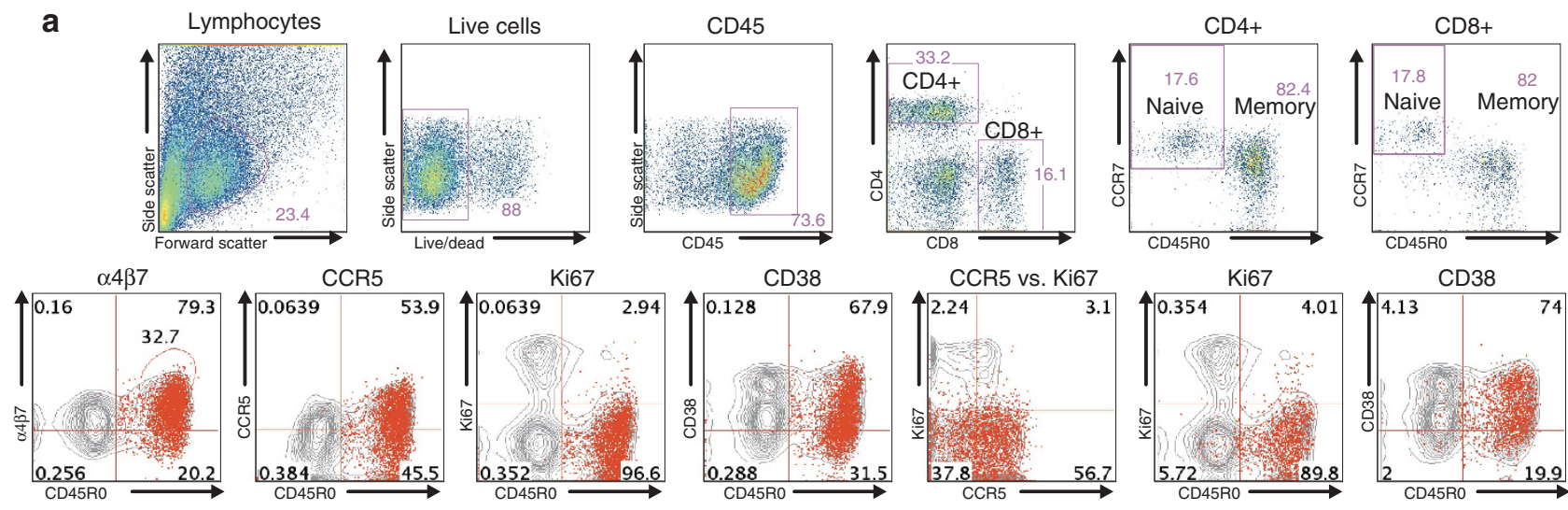

CCR5 vs. Ki67
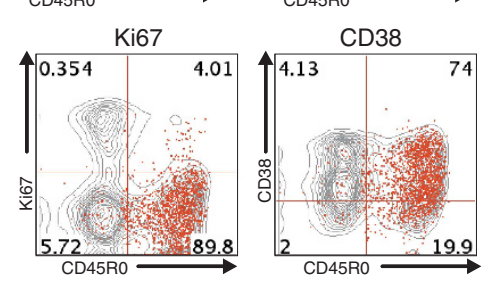

Gated on total memory CD8+ cells

b
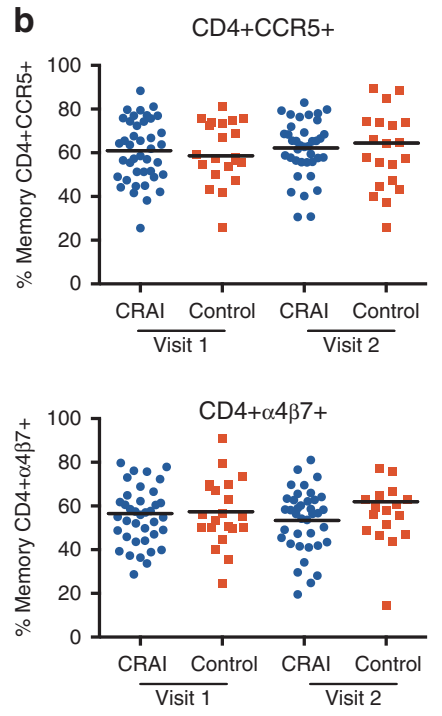
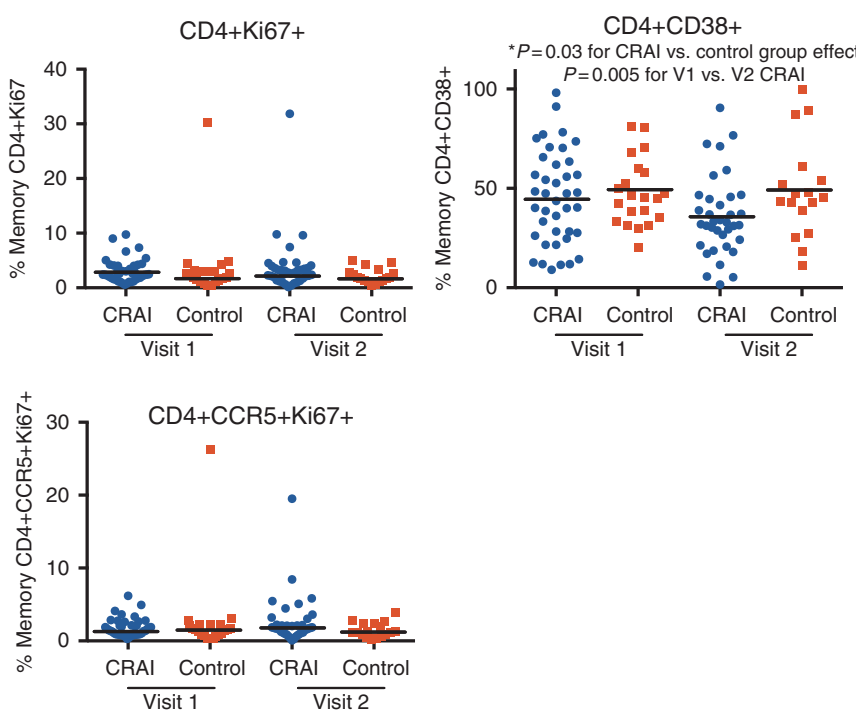

C

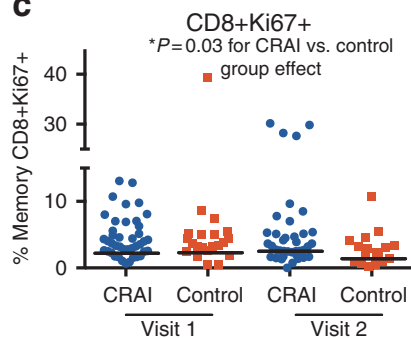

CD8+CD38+

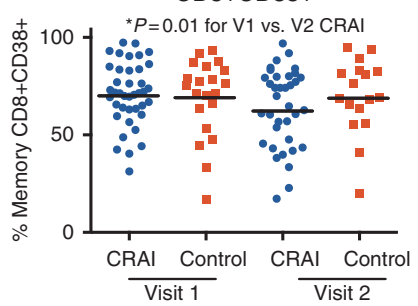

Figure 1 Phenotype of rectal mucosal mononuclear cells (MMCs) shows no differences in HIV target cells between men who have sex with men (MSM) engaging in condomless receptive anal intercourse (CRAI) and controls. (a) Representative gating strategy for rectal MMCs. Lymphocytes were identified by forward and side scatter, then live cells were identified by live/dead staining. CD $45+$ cells were then separated into CD4 + and CD8 + subsets. Memory CD $4+$ and CD8 + cells were identified by excluding CCR7 + CD45RO-cells. Memory CD $4+$ cells were then assessed for expression of $\alpha 4 \beta 7$ (gut homing and HIV co-receptor), CCR5 (HIV co-receptor), Ki67 (proliferation), CD38 (activation), and co-expression of CCR5 and Ki67. Memory CD8 + cells were assessed for expression of Ki67 (proliferation) and CD38 (activation). (b) Results of CD4 + cell phenotyping for MSM engaging in CRAI and controls at visit 1 (abstained from CRAI for $\geqslant 72 \mathrm{~h}$ for MSM engaging in CRAI) and visit 2 (MSM engaged in CRAI $\leqslant 24 \mathrm{~h}$ prior). Black lines represent visit 1 and 2 model-based means as reported in Table 2. (c) Results of CD8 + cell phenotyping for MSM engaging in CRAI and controls at visit 1 and 2 . Black horizontal lines represent visit 1 and 2 model-based means as reported in Table 2.

\section{Rectal CD4 + T cells show greater levels of IL-17 production and CD8 $+\mathrm{T}$ cells show higher levels of pro-inflammatory cytokine expression from MSM engaging in CRAl compared with controls}

We next investigated the function of CD4 + and CD8 + T cells in blood and rectal mucosa with respect to the production of cytokines interferon $\gamma(\mathrm{IFN} \gamma)$, tumor necrosis factor $\alpha(\mathrm{TNF} \alpha)$, and IL-17 following stimulation with phorbol myristate acetate (PMA) and Ionomycin to understand their potential to produce pro-inflammatory cytokines. Rectal CD4 $+\mathrm{T}$ cells from MSM engaging in CRAI stimulated with PMA/Ionomycin showed greater levels of IL-17 production compared with controls (model-based mean 2.4 vs. $1.4 \% ; P=0.01$ ) (Figure 2 and Table 2). Stimulated rectal memory CD8 + T cells from MSM engaging in CRAI showed significantly more secretion of IFN $\gamma$ (model-based mean 56.2 vs. $40.8 \% ; P=0.004$ ) and co-expression of IFN $\gamma$ and TNF $\alpha$ ( 25.3 vs. $15.1 \% ; P=0.004$ ). In addition, MSM who reported higher frequency of CRAI in the month before the baseline visit had greater expression of IFN $\gamma$ (model-based mean $62.9 \%$ vs. $50.8 ; P=0.02$ ) and TNF $\alpha$ (model-based mean $46.1 \%$ vs. $35.9 ; P=0.03$ ) in CD8 + T cells. Enema use was not associated with the cellular differences in cytokine expression. Taken together, the increases in CD4 + IL-17 + cells, the proliferation of CD8 + T cells, and expression of pro-inflammatory cytokines in the gut may indicate a pro-inflammatory mucosal environment in MSM engaging in CRAI. There were no differences in blood cytokine expression in CD4 + or CD8 + cells between MSM engaging in CRAI and controls (see Supplementary Figure 2 and Supplementary Table 1). 
Table 2 Modeling results for rectal mononuclear cell phenotyping (MMC) and functional analyses

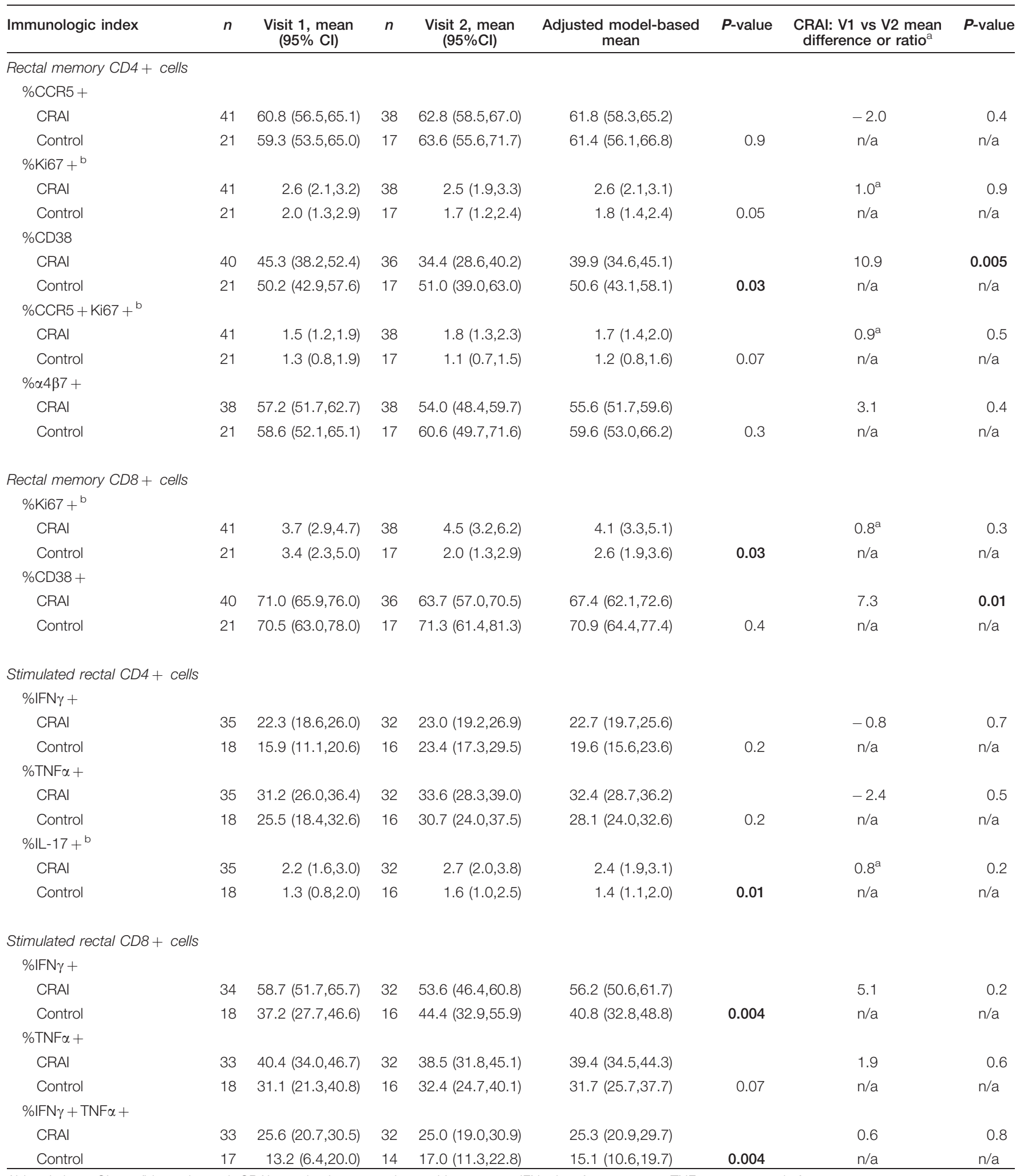

Abbreviations: $\mathrm{Cl}$, confidence interval; CRAl, condomless receptive anal intercourse; IFN $\gamma$, interferon gamma; TNF, tumor necrosis factor.

Results of repeated-measures modeling analysis for rectal MMC phenotyping and intracellular cytokine staining after mitogen stimulation for MSM engaging in condomless receptive anal intercourse (CRAl) and men who never engaged in anal intercourse (controls). MSM abstained from CRAl for $\geq 72 \mathrm{~h}$ prior to visit 1 and engaged in CRAl $\leq 24 \mathrm{~h}$ prior to visit 2. Mixed-effects models controlled for time, time by group interactions, for the laboratory technician performing the assay, age and race. Bolded items are significant $P$-values $(<0.05)$.

${ }^{a}$ Ratio of visit 1 and 2 geometric means reported.

${ }^{\mathrm{b}}$ Report the geometric mean $(95 \% \mathrm{Cl})$. 


\section{Mucosal injury and inflammation are apparent after CRAI in the rectal mucosal transcriptome}

RNA was extracted from rectal biopsies for transcriptome analyses for a subset of MSM engaging in CRAI $(n=18)$ and controls $(n=12)$ from both study visits where samples were available. Three-dimensional plots of the principal components (Figure 3a) showed near overlap of control specimens from visit 1 and 2. There is distinct clustering of the specimens from MSM engaging in CRAI away from the control specimens with further separation visible for the specimens collected within $24 \mathrm{~h}$ of CRAI (visit 2) demonstrating unique rectal mucosal immunologic signatures for MSM engaging in CRAI that are influenced by the timing of the last CRAI episode. a

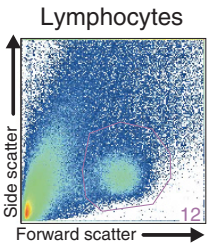

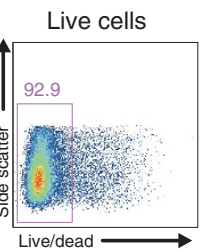
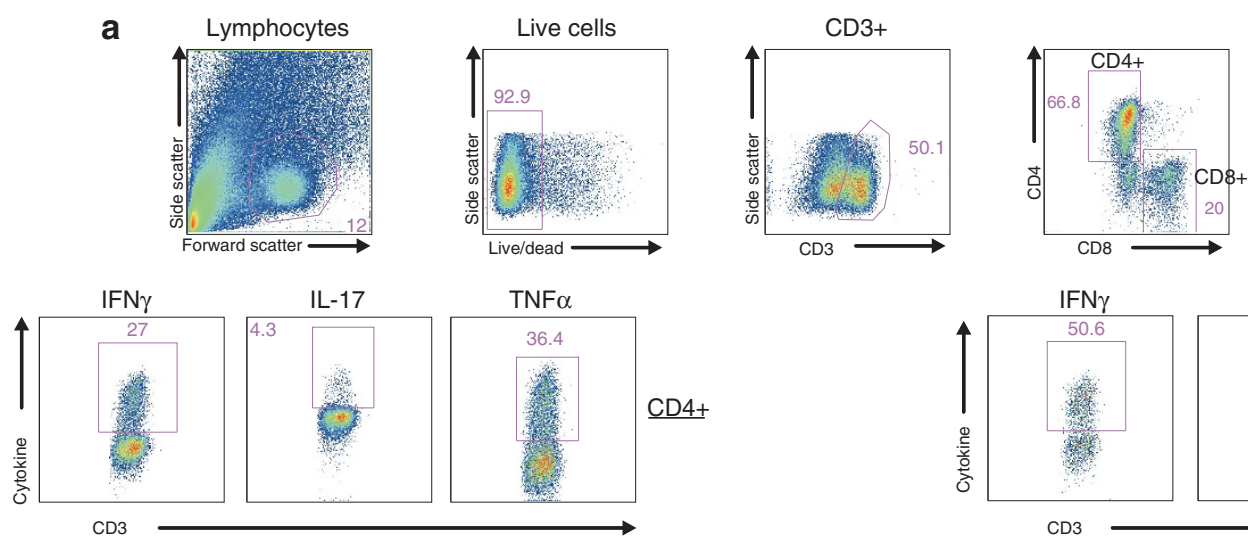

CD3
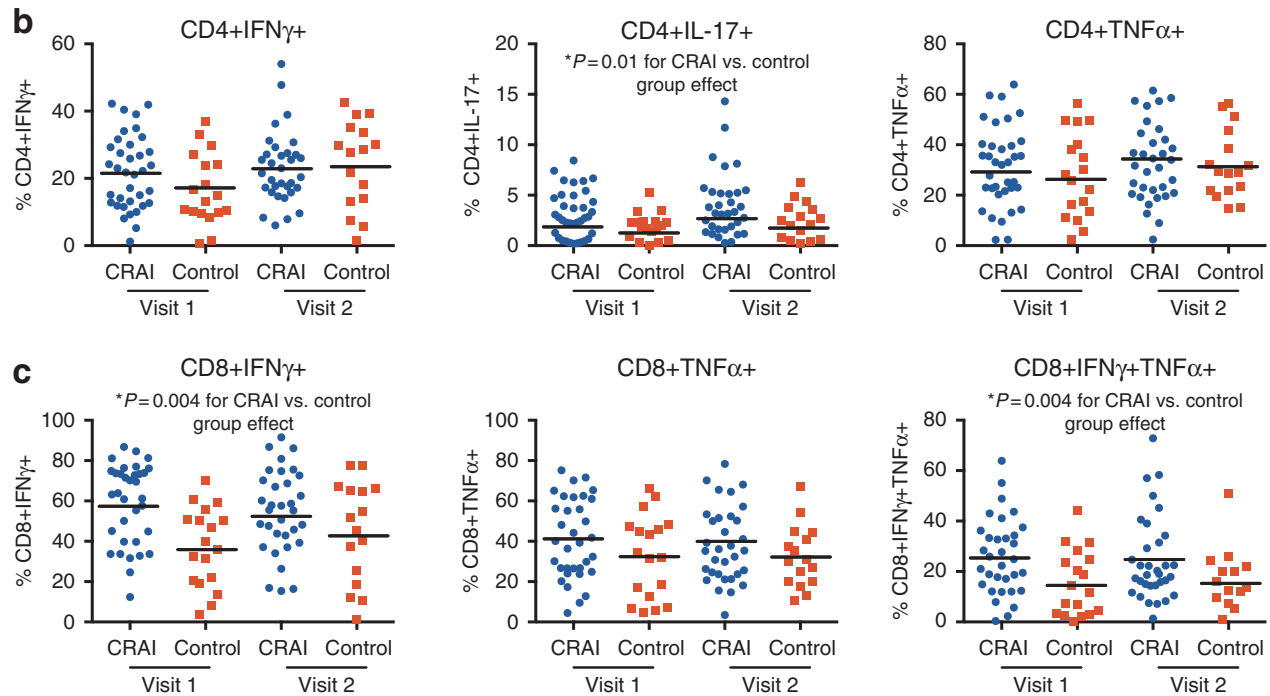

$\mathrm{CD} 8+\mathrm{TNF} \alpha+$
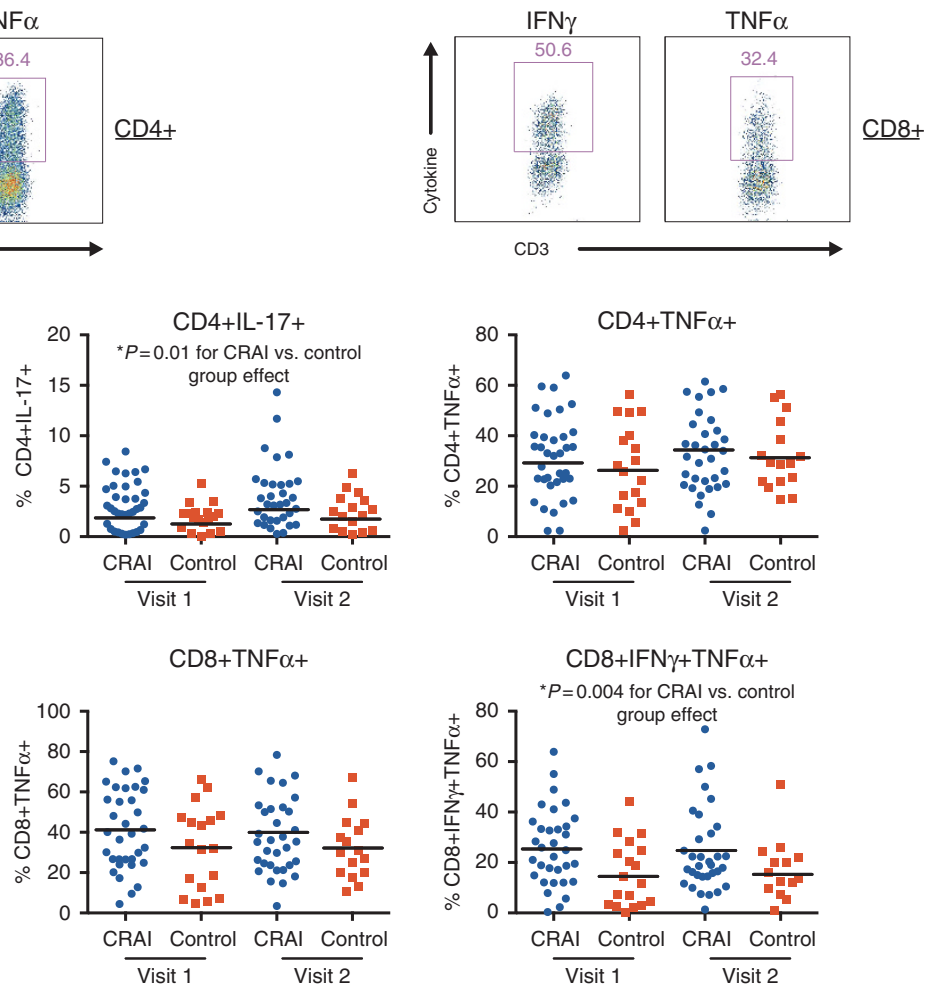

CD3

CD8+IFN $\gamma+$ TNF $\alpha+$

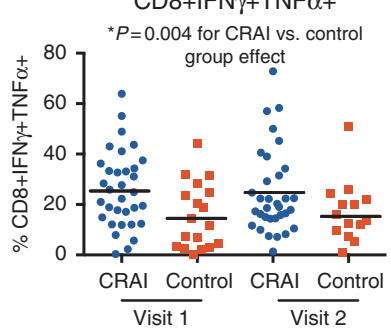

Figure 2 Cytokine production on mitogen stimulation of rectal CD8 + mucosal mononuclear cells (MMCs) shows greater production of proinflammatory cytokines. (a) Representative gating strategy to detect cytokine + CD4 or CD8 T cells. Rectal MMCs were stimulated for $4 \mathrm{~h}$ with PMA/ Ionomycin and stained for indicated cytokines. Lymphocytes were identified by forward and side scatter, then live cells were identified by live/dead staining. CD3 + cells were then separated into CD4 + and CD8 + subsets. Stimulated CD4 + and CD8 + cells were assessed for indicated cytokine production. (b) Summary of cytokine + CD4 T cells for MSM engaging in CRAI and controls at visit 1 and 2. (c) Summary of cytokine + CD8 T cells for MSM engaging in CRAI and controls at visit 1 and 2. Black horizontal lines represent visit 1 and 2 model-based means as reported in Table 2.

Figure 3 RNA-Seq analyses of rectal biopsies shows unique gene signatures among men who have sex with men (MSM) engaging in condomless receptive anal intercourse (CRAI) as compared with controls that differ based on timing of CRAI. (a) Principal components analyses of the rectal mucosal transcriptome of MSM engaging in CRAI. Red ellipse represents MSM who engaged in CRAI $\leqslant 24 \mathrm{~h}$ prior. Blue ellipse represents MSM who abstained from CRAI for $\geqslant 72 \mathrm{~h}$. Green and purple ellipses represent control visits. (b) Venn diagrams depict differentially expressed genes $(q<0.05 ; 0.5>$ fold change > 1.5) comparing control visits (green circle), MSM who abstained from CRAl for at least $72 \mathrm{~h}$ compared with controls (blue circle) and MSM who engaged in CRAI within the last $24 \mathrm{~h}$ compared with MSM who abstained from CRAl for at least $72 \mathrm{~h}$ (red circle). Genes with a fold change $<0.5$ are denoted with *; all other genes had fold change >1.5. (c) Heat map of genes important in granulocyte adhesion and function for MSM engaging in CRAI and controls. The color scales were determined by gene-centric median normalization; blue color denotes downregulation while red color denotes upregulation. (d) Graphic depiction of leading edge genes for pathways of interest identified by gene set enrichment analysis (GSEA) using the Molecular Signatures Database (MSIGDB) program. (e) Heat maps of the leading edge genes from the pathways in (d) (shown as red dots in d) determined to be enriched by GSEA analysis. $q=P$ value adjusted for multiple comparisons. 
a

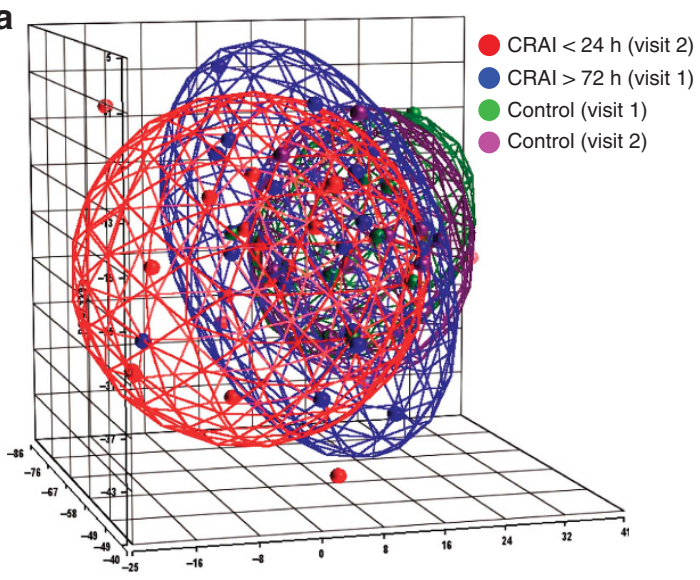

b Differentially expressed genes $(q<0.05,0.5>$ Fold change $>1.5)$

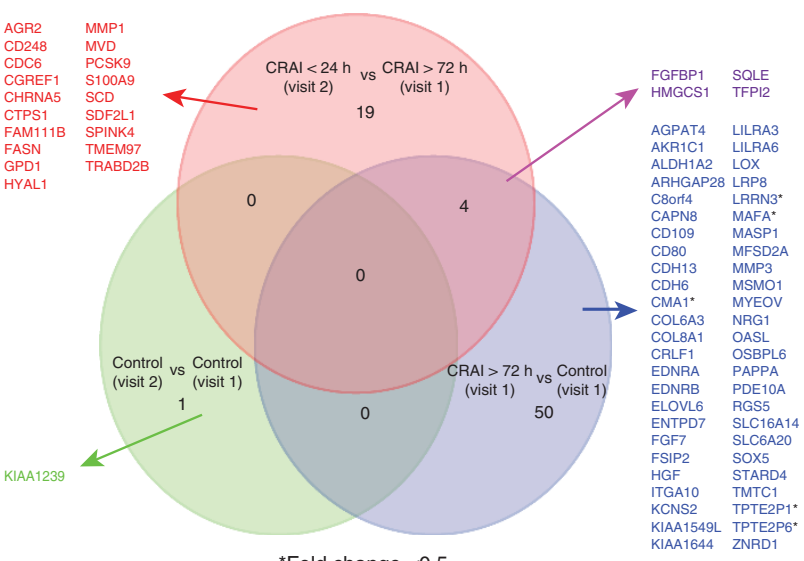

${ }^{*}$ Fold change $<0.5$

c

Granulocyte adhesion and chemokine genes
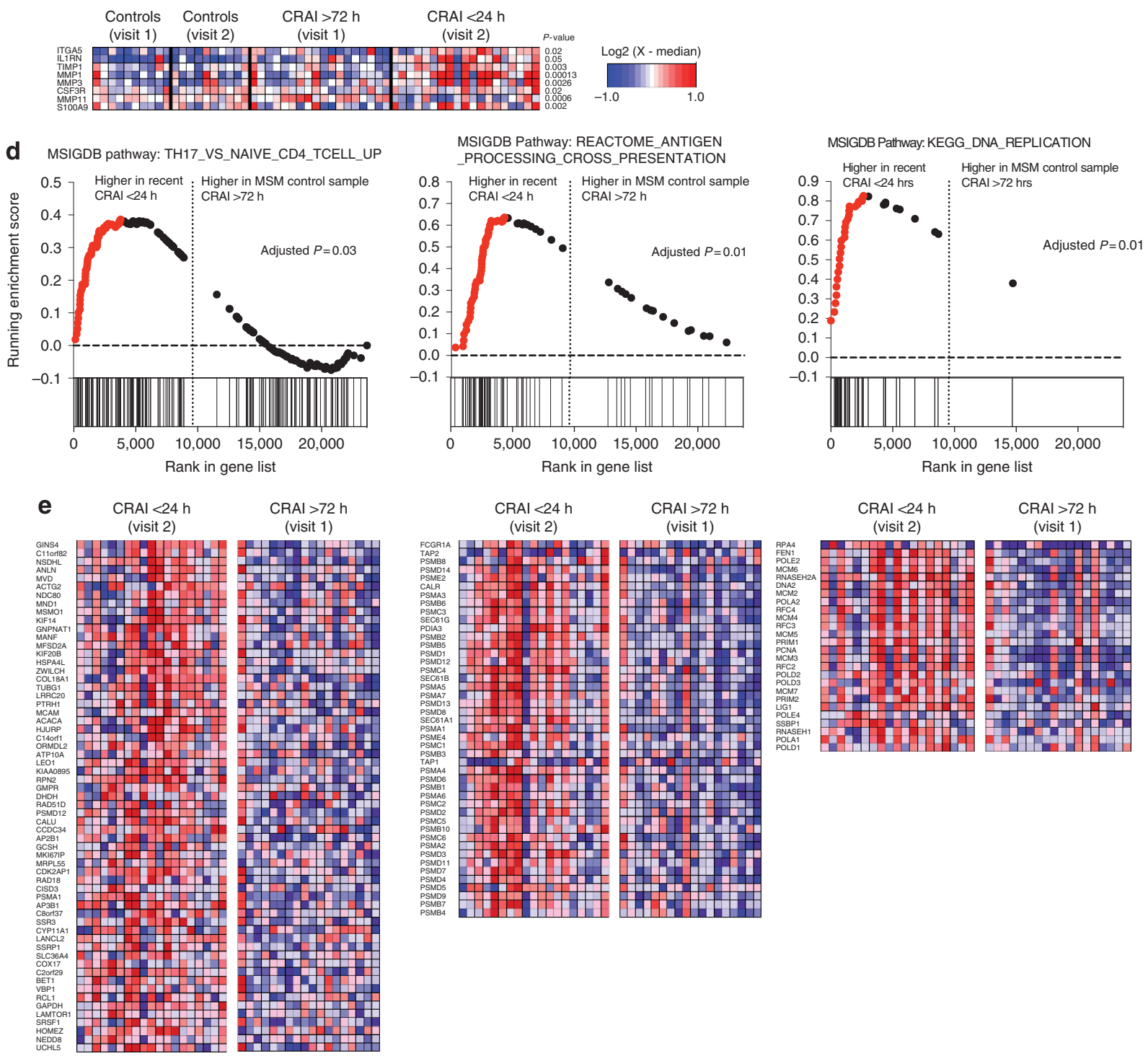
Genes that were significantly different between study groups with a $0.5>$ fold change $>1.5$ threshold and false discovery rate $(q)<0.05$ significance level are shown in Figure 3b. Only 1 gene was significantly different between control specimens at visit 1 and 2 demonstrating limited variation in rectal mucosal gene expression over time in men who do not engage in anal intercourse. Fifty-four genes were significantly different between MSM engaging in CRAI from study visit $1(\geqslant 72 \mathrm{~h}$ from last CRAI) and controls. Genes that were differentially expressed included those implicated in mucosal injury/repair (CAPN8, CAM1, COL6A3, COL8A1, LOX, PAPPA, and $M M P 3$ ), cell proliferation (C8orf4, EDNRB, FGF7, HGF, ZNRD1, and FGFBP1) and immune activation (CD109, CD80, CRLF1, LILRA3, LILRA6, MASP1, and OASL). An additional 19 unique genes were differentially expressed when comparing MSM engaging in CRAI from study visit $2(\leqslant 24 \mathrm{~h}$ from last CRAI) vs. study visit 1 ( $\geqslant 72 \mathrm{~h}$ from last CRAI) and also implicated genes important in mucosal injury/repair (HYAL1 and MMP1), cell proliferation (AGR2, CDC6, CGREF1 and WDR4), and immune activation (S100A9).

We hypothesized that the upregulation of mucosal injury/ repair genes associated with CRAI may be facilitated by neutrophils, a critical mediator of intestinal inflammation. ${ }^{12}$ In fact, we identified several genes important in neutrophil function (ITGA5, IL1RN, TIMP1, MMP1, MMP3, CSF3R, $M M P 11$ and S100A9) that were significantly upregulated in MSM who engaged in CRAI $\leqslant 24 \mathrm{~h}$ prior (Figure $3 \mathrm{c}$ ). Finally, to gain further insight into immunologic pathways that may be associated with recent CRAI, we performed gene set enrichment analysis for enrichment of genes in pathways maintained in the Molecular Signatures Database (MSigDB). For this analysis, we contrasted the transcriptomic data from samples obtained $\leqslant 24 \mathrm{~h}$ post-CRAI with samples obtained $\geqslant 72 \mathrm{~h}$ after CRAI. We screened for significant enrichment of pathways in the C2 (curated genesets), C5 (Gene Ontology genesets), and $\mathrm{C} 7$ (immunologic gene signatures) modules at MSigDB. Gene signatures of interest that were enriched in MSM from study visit 2 ( $\leqslant 24 \mathrm{~h}$ from last CRAI) as compared with study visit 1 ( $\geqslant 72 \mathrm{~h}$ from last CRAI) and controls included signatures for CD4 TH17 vs. naïve CD4 T cells (adjusted $P=0.03$ ), antigen processing and cross-presentation (adjusted $P=0.01$ ), and DNA replication (adjusted $P=0.01$; Figure 3d,e); again implicating injury, repair, and inflammation as important mucosal responses to CRAI.

\section{The rectal mucosal microbiota of MSM engaging in CRAI is enriched for Prevotellaceae, which is associated with markers of mucosal injury and repair}

Results of 16s rRNA sequencing from a mucosal swab collected during rigid sigmoidoscopy showed that MSM engaging in CRAI overall had a lower Shannon Index, a measure of the richness and evenness in a given sample, than controls, which did not differ based on timing of CRAI (model-based mean Shannon Index 5.6 vs. 6.0; $P=0.03$ ). However, beta diversity, which measures the number of shared species between samples by weighted UniFrac distance, did not differ between MSM engaging in CRAI and controls (analysis of similarity (ANOSIM) $R=0.03, P=0.1$ ) or between study visits (ANOSIM $R=0.003, P=0.3$ ). The microbiota of MSM engaging in CRAI was enriched for the family Prevotellaceae (visit 1 median relative abundance 0.12 vs. 0.04 ; visit 2 median relative abundance 0.14 vs. 0.02 ; $P$ value for overall group effect $<0.001)$ compared with controls who were enriched for Bacteroidaceae (visit 1 median relative abundance 0.32 vs. 0.17 ; visit 2 median relative abundance 0.32 vs. $0.15 ; P$ value for overall group effect $=0.003$; Figure 4a,b). However, the enrichment for Prevotellaceae was not uniform among MSM; some MSM were enriched for Bacteroidaceae similar to controls. There were no differences in the relative abundance of the top 10 operational taxonomic units (OTUs) examined between study visits for MSM engaging in CRAI or controls. There were also no associations between the relative abundance of Prevotellaceae or Bacteroidaceae with age, race, enema use, or the frequency of receptive anal intercourse.

Using PICRUSt to predict the metagenomic functional content of the microbiota, the relative abundance of the metabolic pathway associated with arachidonic acid, an important inflammatory intermediate, ${ }^{13}$ metabolism was enriched in the microbiota of MSM (effect size 0.25; $P=0.009)$ and was positively associated with Prevotellaceae abundance (Spearman $r=0.70 ; P<0.0001$; Figure $4 \mathbf{c}, \mathbf{d}$ ). We found no significant associations between the relative abundance of Prevotellaceae and the percentage of rectal mucosal CD4 + cells that expressed CCR5, Ki67, CD38, or IL17 or the percentage of CD8 + cells that expressed Ki67, IFN $\gamma$, or TNF $\alpha$. Finally, we examined associations between the relative abundance of Prevotellaceae and expression of genes associated with mucosal injury/repair among MSM engaging in CRAI at visit 2. Prevotellaceae abundance was associated with ILIRN (Spearman $r=0.58 ; P=0.007$ ), TIMP1 (Spearman $r=0.5 ; \quad P=0.02$ ), and HYAL1 (Spearman $r=0.65$; $P=0.002)$ gene expression in the rectal mucosa, possibly representing a shift in the microbiome to species that are better able to metabolize products of mucosal injury after CRAI (Figure 4d).

\section{DISCUSSION}

In this study of MSM engaging in CRAI and men who do not engage in AI, we found a distinct mucosal phenotype associated with CRAI characterized by expression of genes associated with mucosal injury and repair and neutrophil and Th17 cell activity that were most pronounced after CRAI $\leq 24 \mathrm{~h}$ prior. In addition, we found higher levels of mucosal CD8 + T-cell proliferation and pro-inflammatory cytokine production in the rectal mucosa and a distinct microbiota enriched for Prevotellaceae, which did not differ based on timing of CRAI suggesting that CRAI is also associated with chronic changes in the rectal mucosal immune environment. We hypothesize that mechanical microtrauma occurs during CRAI with deposition of pro-inflammatory semen resulting in transient, subclinical damage of the mucosal epithelium. This, in turn, results in an 



Figure 4 Rectal mucosal microbiota sequencing demonstrates enrichment for Prevotellaceae among men who have sex with men (MSM) engaging in condomless receptive anal intercourse (CRAI) and associations with expression of mucosal injury related genes. (a) Painter plot of the top 10 operational taxonomic units (OTUs) represented in rectal mucosal samples from MSM engaging in CRAl and controls. (b) Results of repeated measures modeling analysis for the top 10 OTUs identified for MSM engaging in CRAI and men who never engaged in anal intercourse (controls). Mixed effects models controlled for time, time by group interactions, age, and race. MSM abstained from CRAI for $\geqslant 72 \mathrm{~h}$ before visit 1 and engaged in CRAI $\leqslant 24 \mathrm{~h}$ before visit 2 . (c) PICRUSt analysis to predict the metagenomic functional content from visit 2 specimens for MSM engaging in CRAI and controls. (d) The relative abundance of Prevotellaceae was significantly associated with the arachidonic acid metabolism pathway as well as several mucosal injury and repair associated genes: TIMP1, HYAL1, and IL $1 R N$. V1 = visit $1 ; \mathrm{V} 2=$ visit 2 ; *adjusted $P$ value $\leqslant 0.01$; **adjusted $P$ value $\leqslant 0.05$ for Wilcoxon rank-sum test for differences in median expression between MSM and controls; $r_{\mathrm{s}}=$ Spearman rank correlation coefficient.

inflammatory response and exposure of the lamina propria to the gut commensal microbiota and subsequent mucosal healing. Over time, the microbiota shifts to organisms better able to metabolize products of mucosal injury, and an adaptive pro-inflammatory CD4 + and CD8 $+\mathrm{T}$ cell immune response develops. For HIV-negative MSM, this is the first description of the immune context of the rectal mucosa in which HIV transmission might occur after exposure or in which a biomedical prevention intervention (e.g., a microbicide or vaccine) should exert its effect.

The physiological response to mucosal injury, recently reviewed by Leoni et al, ${ }^{14}$ is generally characterized by initial inflammation mediated by infiltration of innate immune cells (e.g., neutrophils and macrophages) which secrete proteases, cytokines, and chemokines, followed by proliferation of epithelial cells, and remodeling of the extracellular matrix. Our data show findings consistent with mucosal injury, though likely subclinical, after CRAI. The rectal mucosal transcriptome $24 \mathrm{~h}$ after CRAI showed upregulation of genes important in tissue remodeling, neutrophil function, as well as DNA proliferation and antigen presentation, and cross-presentation signatures. Neutrophil activity and neutrophil-associated proteins in the female ${ }^{15,16}$ and male genital tract ${ }^{17}$ have been associated with susceptibility to HIV infection, and reduced neutrophil activation has been associated with the highly exposed seronegative phenotype. ${ }^{18}$ The contribution of neutrophils to HIV susceptibility may be mediated by epithelial barrier disruption by neutrophil secreted proteases and/or 
increased HIV target cell recruitment ${ }^{19}$ and deserves further investigation to understand their role in rectal HIV transmission. In addition, recent data suggest that infiltrating neutrophils after CRAI could be exploited in HIV vaccine strategies that produce non-neutralizing antibodies for their Fc receptormediated phagocytosis capabilities. ${ }^{20}$

Although we did not show an increase in activated CD4 $+\mathrm{T}$ cells or increased HIV co-receptor expression among MSM engaging in CRAI, our data do show higher levels of Th17 cells, more proliferating CD8 $+\mathrm{T}$ cells, and greater production of IFN $\gamma$ and $\mathrm{TNF} \alpha$ in CD8 $+\mathrm{T}$ cells among MSM engaging in CRAI. Th17 gene signatures were also upregulated within $24 \mathrm{~h}$ of CRAI, and it is likely that Th17 cells are a key mediator of the response to CRAI. Th17 cells play a dichotomous role in the gastrointestinal mucosa, which is yet to be fully elucidated. ${ }^{21}$ Th17 cells are critical for maintenance of mucosal epithelial barrier function but have also been shown to contribute to a colitogenic state. ${ }^{22}$ Th17 cells also recruit neutrophils to sites of mucosal injury further supporting their likely contribution to the mucosal response to CRAI. ${ }^{23}$ On the other hand, the Th17 cell subset is highly susceptible to HIV infection. ${ }^{24,25}$ Therefore, the response to CRAI may be promoting a cellular composition in the mucosal tissues that facilitates HIV transmission. In addition, pro-inflammatory $\mathrm{CD} 8+\mathrm{T}$ cells have been implicated in the pathogenesis of inflammatory bowel disease, which is characterized by gut mucosal lesions, mucosal inflammation and damage, and microbial translocation; and it is plausible that a similar, though certainly less severe and non-pathologic, mucosal immune response is seen after CRAI. ${ }^{26}$

An additional critical determinant of mucosal immune defense is the composition of the microbiota ${ }^{27}$; and a diverse gut microbiome dominated by the phyla Bacteroidetes and Firmicutes is typical in healthy adults that can vary significantly in relative abundance between individuals. ${ }^{28}$ Variations in the gastrointestinal microbiota can be seen with changes in diet, antimicrobial use, stress, and environmental exposures. ${ }^{29}$ The microbiome of the female genital tract is altered after exposure to semen, ${ }^{30}$ which carries its own microbiome distinct in composition from the intestinal microbiome, ${ }^{31}$ and it is biologically plausible that CRAI results in alteration of the rectal mucosal microbiome. Our data agree with a recent study showing that the stool microbiota of MSM was enriched for Prevotella species as opposed to non-MSM that was enriched for Bacteroides, although the population studied in this report was heavily weighted toward HIV-positive individuals. ${ }^{32}$ Our data extend this finding to the mucosally adherent bacterial populations in the rectum and also question previous reports that have attributed an observed enrichment of Prevotellaceae to HIV infection since all men included in our study were healthy and HIV negative. ${ }^{33}$ Nonetheless, it is notable that the rectal mucosal microbiota was stable over time, and that the microbiota of several MSM engaging in CRAI was similar to controls indicating that this enrichment for Prevotellaceae is not uniform. We did not control for diet in our study; however, the previously published work did not find an association with diet and the relative abundance of Prevotellaceae, and the stool samples used in those studies were collected from MSM in Spain and Scandinavia. Therefore, the consistent findings of enrichment of Prevotellaceae in MSM seen in three different geographic regions suggest that diet is not the cause of this finding.

The enrichment of Prevotellaceae in MSM engaging in CRAI was closely associated with the increased relative abundance of the arachidonic acid metabolism pathway in the predicted metagenomic functional content. Interestingly, we also found several associations between the relative abundance of Prevotellaceae and genes, such as HYAL1, implicated in tissue injury and repair. Inflammatory cells generally contain arachidonic acid, the major substrate for eicosanoids (e.g., prostaglandins, thromboxanes, and leukotrienes), which are important in modulating the intensity and duration of the inflammatory response. ${ }^{13}$ Therefore, our data suggest that the mucosal inflammatory cascade that occurs after CRAI could lead to a shift in the microbiota to species better able to metabolize the products of mucosal injury rather than a mediator of mucosal inflammation as has been suggested previously. ${ }^{33}$ Interestingly, an increase in the relative abundance of Prevotella after mucosal injury has been reported in the mouse model and further supports our data. ${ }^{34}$ It is also notable that Prevotella species are present in seminal fluid and may contribute to this finding. ${ }^{31}$ Of interest, we did not find any significant association between the relative abundance of Prevotellaceae and the cellular milieu of the rectal mucosa; therefore, further studies will be necessary to determine whether the composition of the rectal mucosal microbiota can influence susceptibility to HIV infection. Nonetheless, the composition of the microbiota may be an important variable to consider in the design of candidate HIV vaccines particularly for MSM at-risk of HIV infection, and therapeutic manipulation of the microbiota could potentially be utilized as adjunctive therapy to optimize mucosal immune responses. ${ }^{35}$

Our study is limited in that our sample size is modest, and we did not examine the effects of CRAI without semen exposure. Inclusion of receptive anal intercourse with condom use time points will be an important consideration for future studies. Currently, there is no acceptable biomarker for semen exposure in the rectum (e.g., prostate specific antigen testing); ${ }^{36,37}$ therefore, we were unable to validate self-reported CRAI activity in our cohort. We did not control for diet or other environmental factors in the microbiome analyses, and unmeasured confounding factors may explain the enrichment for Prevotellaceae among MSM. Innate immune cellular responses were not examined by flow cytometry. Immunohistochemistry assays are ongoing to examine differences in innate cells and in the tissue distribution of HIV target cell populations that may reveal additional effects of CRAI not apparent with flow cytometry. Rectal mucus specimens were not collected to assess secreted factors that are important in mucosal defenses and will need to be considered 
in future studies. Finally, our findings are generally descriptive in nature, and further experiments (e.g., colorectal explant challenges) or large, longitudinal cohort studies with HIV acquisition endpoints will be necessary to determine the relevance of our mucosal findings for rectal HIV susceptibility among MSM.

In summary, we have identified a unique immunologic milieu in the rectum of MSM engaging in CRAI characterized by cellular markers of inflammation, molecular signatures of mucosal injury, and repair, and an enrichment of the microbiota for Prevotellaceae. It is unclear, at this time, whether these factors could influence susceptibility to HIV infection or mucosal immune response to vaccines and further studies will be necessary. Nonetheless, this is the most comprehensive evaluation of the immune environment of the rectal mucosa in HIV negative at-risk MSM to date, and this valuable data will be useful to the design of future mechanistic studies of HIV transmission and biomedical prevention intervention studies.

\section{METHODS}

The clinical cohort. HIV-negative MSM aged 18-45 years in good health who were engaging in CRAI with an HIV-negative partner were recruited from the Atlanta community. HIV-negative men aged 18-45 years in good health who had never engaged in anal intercourse, regardless of sexual orientation, were recruited as controls. Inclusion criteria for MSM engaging in CRAI included HIV-negative status, being in a monogamous relationship for $\geqslant 45$ days with an HIV-negative man, and reporting a minimum of four episodes of CRAI in the last month. MSM engaging in CRAI were also asked to keep an electronic or paper sex diary for the duration of the study that recorded each episode of receptive anal intercourse, whether a condom was used, whether ejaculation occurred during penetrative intercourse, type of lubricant used (if any), and type of enema used (if any). Men in both study groups who were determined by the principal investigator to be high risk for rectal biopsy procedures due to medical comorbidities or who intended to take pre-exposure prophylaxis during the study were not enrolled.

The study consisted of three visits. During the screening study visit, eligible participants provided informed consent, underwent a brief sexual and medical history and physical examination, rapid HIV testing, and blood collection. The next study visit occurred 1-6 weeks later (median 2.6 weeks) and participants underwent peripheral blood and rectal biopsy sampling via rigid sigmoidoscopy with no prior bowel preparation (visit 1). All biopsies were collected $\sim 3-10 \mathrm{~cm}$ from the anal verge. MSM who were engaging in CRAI were asked to abstain from CRAI for $\geqslant 72 \mathrm{~h}$ before study visit 1 . The final study visit occurred 8-16 weeks after study visit 1 (median 9 weeks) and participants again underwent peripheral blood and rectal biopsy sampling (visit 2). The intestinal epithelium is the most rapidly selfrenewing tissue in the human body, and when injury occurs, it is quickly repaired. ${ }^{38,39}$ We hypothesized that some effects of CRAI may be transient; therefore, MSM engaging in CRAI were asked to engage in $\mathrm{CRAI} \leqslant 24 \mathrm{~h}$ before study visit 2 . The Institutional Review Board (IRB) at Emory University approved this study.

Blood and rectal mucosal mononuclear cell phenotyping. Blood collected in EDTA tubes was processed by Ficoll density gradient to separate peripheral blood mononuclear cells, and 5 pinch biopsies from the rectal mucosa were processed by collagenase digestion to separate mucosal mononuclear cells as described previously. ${ }^{40}$ A complete list of antibodies used for phenotyping is available in the Supplementary Materials. Mononuclear cells isolated from the blood and rectal biopsies were stained with LIVE/DEAD Near-IR Dead Cell stain (Life Technologies) at room temperature for $15 \mathrm{~min}$ in PBS to stain for dead cells. Cells were then washed with fluorescenceactivated cell sorting (FACS) wash and stained on the surface using antibodies specific to CD3, CD4, CD8, CCR7, CD45RO, CD45, CCR5, $\alpha 4 \beta 7, \quad$ CD38, HLA-DR and then treated with $1 \times$ BD FACS Lysing solution for $10 \mathrm{~min}$ at room temperature, permeabilized with $1 \times \mathrm{BD}$ permeablizing solution for $10 \mathrm{~min}$ at room temperature, washed with FACS wash, stained with anti-Ki67 antibodies, washed $2 \times$ with FACS wash, and acquired on the LSR-Fortessa platform and analyzed with Flowjo software (Treestar Inc. CA). Staining for HLA-DR was suboptimal during the study; therefore, further analyses of this antibody were not performed.

Intracellular cytokine staining. One million cells were stimulated with $25 \mathrm{ng} \mathrm{ml}^{-1}$ of PMA and $500 \mathrm{ng} \mathrm{ml}^{-1}$ of Ionomycin in the presence of Brefeldin A ( $5 \mu \mathrm{g} \mathrm{ml}^{-1}$; Sigma-Aldrich; St. Louis, MO) and Golgi stop $\left(0.5 \mu \mathrm{l} \mathrm{ml}^{-1}\right.$; BD Pharmingen; San Jose, CA) and incubated for $4 \mathrm{~h}$ at $37^{\circ} \mathrm{C}$ in the presence of $5 \% \mathrm{CO}_{2}$. At the end of stimulation, cells were placed at $4^{\circ} \mathrm{C}$ overnight. Cells were then washed once with FACS wash and surface stained with anti-CD3, anti-CD4, and antiCD8 antibodies at $4{ }^{\circ} \mathrm{C}$ for $20 \mathrm{~min}$. Next, cells were washed once with FACS wash, fixed with cytofix/cytoperm (BD Pharmingen) for $20 \mathrm{~min}$ at $4{ }^{\circ} \mathrm{C}$ and washed with $1 \times$ Perm wash (BD Pharmingen). Cells were then incubated for $30 \mathrm{~min}$ at $4{ }^{\circ} \mathrm{C}$ with antibodies specific to IL-17A, IFN $\gamma$, TNF $\alpha$, and CD4, washed once with Perm wash, once with FACS wash, and re-suspended in PBS containing 1\% formalin. Cytokine secretion from unstimulated and stimulated cells was assessed by flow cytometry. If we were unable to acquire at least 100 CD4 or CD8 cells from the stimulated specimens or if there was a lack of cellular response for all of the cytokines, the specimen was classified as a non-response to stimulation and excluded from all further analyses.

Transcriptome sequencing. RNA-Seq analyses were conducted at the Yerkes NHP Genomics Core on a subset of MSM engaging in CRAI $(n=18)$ and controls $(n=12)$. Total RNA was extracted from rectal pinch biopsies using QIAGEN RNEAsy Mini kits, RNA quality assessed using Agilent Bioanalyzer analysis. Polyadenylated transcripts were purified on oligo-dT magnetic beads, reverse transcribed using random hexamers, fragmented, and incorporated into barcoded complementary DNA libraries based on the Illumina TruSeq platform. Libraries were validated by microelectrophoresis, pooled, and sequenced on an Illumina HiSeq 1000 (101 bp) to an average read depth of $18.4 \mathrm{M}^{41}$ Reads were aligned to human RefSeq hg19 reference using STAR software (v2.3.0e) (http://code.google.com/p/rna-star/). ${ }^{42}$ One sample from a subject diagnosed with Chlamydia at the time of rectal biopsy collection was excluded from the analysis. The RNA-Seq data were deposited to the GEO database (accession GSE83245).

Microbiota sequencing. Swabs of the rectal mucosa were collected from a subset of MSM engaging in CRAI $(n=34)$ and controls ( $n=17$ ) through the sigmoidoscope at $\sim 8-10 \mathrm{~cm}$ from the anal verge and stored at -80 in lysis buffer. DNA was extracted, amplified, and sequenced using methods described previously. ${ }^{43}$ Reads were trimmed (Q20) and adapter sequences removed by means of FastQ-mcf (https://code.google.com/archive/p/ea-utils/wikis/FastqMcf.wiki).

Overlapping regions of $16 \mathrm{~S}$ rRNA reads were assembled into contigs using PEAR (ref. 44) and analyzed with QIIME 1.9.1. (ref. 45) $16 \mathrm{~S} \mathrm{V1-V2} \mathrm{contigs} \mathrm{were} \mathrm{clustered} \mathrm{into} \mathrm{OTUs} \mathrm{by} \mathrm{means} \mathrm{of} \mathrm{a} \mathrm{sub-}$ sampled open reference picking algorithm ${ }^{46}$ using Sortmerna (reference picking) ${ }^{47}$ and Sumaclust (de novo OTU picking at $97 \%$ sequence similarity; https://metabarcoding.org/sumatra). OTU singletons were removed and, OTU centroids aligned using PyNAST (ref. 48) and subsequent phylogenetic trees were inferred by FastTree. ${ }^{49}$ We rarefied each sample to 2,215 OTUs, a sufficient quantity to cover full microbial diversity based on rarefaction curves 
for each sample. Centroid sequences from each OTU were aligned against the Greengenes database (version 13-8) for taxonomy assignment. ${ }^{50}$ The metabolic content of the metagenomes was predicted by means of PICRUSt. ${ }^{51}$ PICRUSt output was then analyzed using STAMP. ${ }^{52}$ Raw 16 s rRNA sequences were placed in the NCBI Sequence Read Archive (SRA) under BioProject PRJNA322688.

Statistical analyses. Demographic and clinical characteristics were compared between study groups (MSM engaging in CRAI or controls) with Wilcoxon rank-sum test for continuous variables and with Fisher's exact test for proportions. To assess longitudinal outcomes between MSM engaging in CRAI and controls, repeated-measures analyses for cellular phenotyping and stimulation outcomes from blood and mucosal mononuclear cells were performed with a means model via the SAS MIXED Procedure (version 9.4) providing separate estimates of the means by time on study (visit 1 and 2) and study group (MSM engaging in CRAI or controls). The model included six predictors (study group, time on study, the statistical interaction between study group and time on study, technician performing the assay, age, and race). If the outcome in the two study groups was consistently different or similar (i.e., no interaction) then the main effect test for study group was used as the primary test of study group differences. Additionally a paired $t$ test was used to detect differences between visit 1 and 2 within the MSM engaging CRAI study group within the framework of the mixed effects linear model. We conducted sensitivity analyses excluding a few specimens from men diagnosed with an STI during the biopsy visits and results were not changed. Associations between enema use and frequency of CRAI on cellular phenotyping and stimulation outcomes were also examined with mixed models between MSM engaging in CRAI and controls after covariate adjustment. Use of lubricants could not be examined given that almost all MSM engaging in CRAI reported lubricant use. All statistical tests were two-sided and unadjusted for multiple comparisons and should be interpreted with caution. Due to small sample sizes, results from these studies also focused on magnitude of the differences for each outcome, consistency of findings, and biological and clinical significance.

RNA-Seq statistical analyses. To examine differential gene expression in rectal biopsy tissues generated by RNA-Seq, estimates of gene-wise and isoform-wise expression levels for individual genes were performed using DESeq, which normalizes gene expression level estimates across samples and also corrects for nonuniformity in read distributions across each gene. ${ }^{53}$ Clustering by covariance PCA and visualization (i.e., heat maps) of expression data were performed in Partek Genomics Suite software (Partek Inc., St Louis, MO, USA). Differentially expressed genes were analyzed for enriched gene families/pathways/ protein interactions using Gene Set Enrichment Analysis with the Immunological Signatures Databases hosted at the MSigDB database (www.broadinstitute.org/gsea/msigdb/collection.jsp) and with custom gene-sets generated from in-house data sets. Gene set enrichment analysis was performed on the regularized $\log$ (rlog) expression table produced by DESeq2 employing a weighted enrichment statistic and Signal2Noise as the ranking metric and using 1000 phenotype permutations.

Microbiome statistical analyses. After taxonomic assignment, the alpha diversity was measured using the Shannon Index and statistical significance was assessed with Monte Carlo simulations. Beta diversity was measured using the weighted UniFrac distance, ${ }^{54}$ and visualized by principal coordinates plots. A global test of the differences in the microbial composition between the groups was conducted using the ANOSIM test. ${ }^{55}$ Next, to compare the diversity and relative abundance of a specific bacterial family (i.e., an individual taxon) between the two groups, we treated the family abundance as a continuous variable and conducted repeated measures analyses using linear mixed models controlling for age and race as described above for the Shannon Index value and the top 10 OTUs identified.
Finally, in exploratory analyses, Spearman rank correlation coefficients were examined between the visit 2 relative abundance of specific bacterial families of interest (Prevotellaceae and Bacteroidaceae), flow cytometry and cell stimulation values of interest $(\mathrm{CD} 4+\mathrm{CCR} 5+, \mathrm{CD} 4+\mathrm{CD} 38+, \mathrm{CD} 4+\mathrm{Ki} 67+, \mathrm{CD} 4+\mathrm{IL}-17+$, $\mathrm{CD} 8+\mathrm{Ki} 67+, \mathrm{CD} 8+\mathrm{IFN} \gamma, \mathrm{CD} 8+\mathrm{TNF} \alpha+)$, and the normalized read count generated from RNA-Seq for mucosal injury/repair genes of interest (IL1RN, ITGA5, TIMP1, MMP11, CSF3R, S100A9, MMP1, MMP3, HYAL1, and CMA1).

Data availability. The RNA-Seq read and expression data have been deposited in NCBI's Gene Expression Omnibus and are accessible through GEO Series accession number GSE83245. Raw 16s rRNA sequences were placed in the NCBI Sequence Read Archive (SRA) under the associated BioProject PRJNA322688.

SUPPLEMENTARY MATERIAL is linked to the online version of the paper at http://www.nature.com/mi

\section{ACKNOWLEDGMENTS}

We thank the study volunteers for their participation in this research. K23 Al108335 (C.F.K.), U19 Al109633 (R.R.A.), The Emory Center for AIDS Research P30AI050409, The Atlanta Clinical and Translational Science Institute UL1TR000454.

\section{DISCLAIMER}

The findings and conclusions in this paper are those of the authors and do not necessarily represent the views of Emory University or the Centers for Disease Control and Prevention.

\section{AUTHOR CONTRIBUTIONS}

C.F.K. conceived the study, performed data analyses, and wrote the manuscript. C.S.K. performed laboratory assays, data analyses, and provided critical review of the manuscript. T.J.B.D. performed data analyses, wrote, and provided critical review of the manuscript. C.D. performed laboratory assays and data analyses. H.W.L. performed laboratory assays and data analyses. J.Y. performed data analyses and provided critical review of the manuscript. K.A.E. assisted with the design of the study, performed data analyses, and wrote and provided critical review of the manuscript. GKT performed data analyses and provided critical review of the manuscript. M.J.M. assisted with enrollment of the clinical cohort and provided critical review of the manuscript. P.S.S. provided assistance with recruitment and enrollment of the clinical cohort and provided critical review of the manuscript. S.E.B. performed laboratory assays, data analyses, wrote, and provided critical review of the manuscript. R.R.A. oversaw the conduct of the laboratory assays, data analyses, and provided critical review of the manuscript.

\section{DISCLOSURE}

The authors declare no conflict of interest.

c) 2017 Society for Mucosal Immunology

\section{REFERENCES}

1. Centers for Disease Control and Prevention. HIV Surveillance Report, 2014. 26 (2015) <http://www.cdc.gov/hiv/library/reports/surveillance/ $\% 3 \mathrm{E}$.

2. US Public Health Service. Pre-exposure Prophylaxis for the Prevention of HIV Infection in the United States-2014: A Clincal Practice Guideline (2014). Available at: http://www.cdc.gov/hiv/pdf/prepguidelines2014.pdf.

3. Sullivan, P.S., Salazar, L., Buchbinder, S. \& Sanchez, T.H. Estimating the proportion of HIV transmissions from main sex partners among men who have sex with men in five US cities. AIDS 23, 1153-1162 (2009).

4. Hladik, F. \& McElrath, M.J. Setting the stage: host invasion by HIV. Nat. Rev. Immunol. 8, 447-457 (2008).

5. Sharkey, D.J., Tremellen, K.P., Jasper, M.J., Gemzell-Danielsson, K. \& Robertson, S.A. Seminal fluid induces leukocyte recruitment and cytokine 
and chemokine mRNA expression in the human cervix after coitus. J. Immunol. 188, 2445-2454 (2012).

6. Haase, A.T. Targeting early infection to prevent HIV-1 mucosal transmission. Nature 464, 217-223 (2010).

7. Mowat, A.M. \& Viney, J.L. The anatomical basis of intestinal immunity. Immunol. Rev. 156, 145-166 (1997).

8. Poles, M.A., Elliott, J., Taing, P., Anton, P.A. \& Chen, I.S. A preponderance of CCR5 $(+) \operatorname{CXCR} 4(+)$ mononuclear cells enhances gastrointestinal mucosal susceptibility to human immunodeficiency virus type 1 infection. J. Virol. 75, 8390-8399 (2001).

9. McElrath, M.J. et al. Comprehensive assessment of HIV target cells in the distal human gut suggests increasing HIV susceptibility toward the anus. J. Acquir. Immune Defic. Syndr. 63, 263-271 (2013).

10. Baggaley, R.F., White, R.G. \& Boily, M.C. HIV transmission risk through anal intercourse: systematic review, meta-analysis and implications for HIV prevention. Int. J. Epidemiol. 39, 1048-1063 (2010).

11. Cicala, C., Arthos, J. \& Fauci, A.S. HIV-1 envelope, integrins and coreceptor use in mucosal transmission of HIV. J. Transl. Med. 9 (Suppl 1), S2 (2010).

12. Fournier, B.M. \& Parkos, C.A. The role of neutrophils during intestinal inflammation. Mucosal Immunol. 5, 354-366 (2012).

13. Calder, P.C. n-3 polyunsaturated fatty acids, inflammation, and inflammatory diseases. Am. J. Clin. Nutr. 83, 1505S-1519S (2006).

14. Leoni, G., Neumann, P.A., Sumagin, R., Denning, T.L. \& Nusrat, A. Wound repair: role of immune-epithelial interactions. Mucosal Immunol. 8, 959-968 (2015).

15. Levinson, P. et al. Levels of innate immune factors in genital fluids: association of alpha defensins and LL-37 with genital infections and increased HIV acquisition. AIDS 23, 309-317 (2009).

16. Birse, K. et al. Molecular signatures of immune activation and epithelial barrier remodeling are enhanced during the luteal phase of the menstrual cycle: implications for HIV susceptibility. J. Virol. 89, 8793-8805 (2015).

17. Hirbod, T. et al. HIV acquisition is associated with increased antimicrobial peptides and reduced HIV neutralizing IgA in the foreskin prepuce of uncircumcised men. PLoS Pathog. 10, e1004416 (2014).

18. Hernandez, J.C., Giraldo, D.M., Paul, S. \& Urcuqui-Inchima, S. Involvement of neutrophil hyporesponse and the role of Toll-like receptors in human immunodeficiency virus 1 protection. PLoS One 10, e0119844 (2015).

19. Arnold, K.B. et al. Increased levels of inflammatory cytokines in the female reproductive tract are associated with altered expression of proteases, mucosal barrier proteins, and an influx of HIV-susceptible target cells. Mucosal Immunol. 9, 194-205 (2016).

20. Sips, M. et al. Fc receptor-mediated phagocytosis in tissues as a potent mechanism for preventive and therapeutic HIV vaccine strategies. Mucosal Immunol. 9, 1584-1595 (2016).

21. O'Connor, W. Jr, Zenewicz, L.A. \& Flavell, R.A. The dual nature of $T(H) 17$ cells: shifting the focus to function. Nat. Immunol. 11, 471-476 (2010).

22. Korn, T., Bettelli, E., Oukka, M. \& Kuchroo, V.K. IL-17 and Th17 cells. Annu. Rev. Immunol. 27, 485-517 (2009).

23. Pelletier, M. et al. Evidence for a cross-talk between human neutrophils and Th17 cells. Blood 115, 335-343 (2010).

24. Rodriguez-Garcia, M., Barr, F.D., Crist, S.G., Fahey, J.V. \& Wira, C.R. Phenotype and susceptibility to HIV infection of CD4+ Th17 cells in the human female reproductive tract. Mucosal Immunol. $\mathbf{7}$, 1375-1385 (2014).

25. Monteiro, P. et al. Memory CCR6 + CD4 + T cells are preferential targets for productive HIV type 1 infection regardless of their expression of integrin beta7. J. Immunol. 186, 4618-4630 (2011).

26. Boschetti, G. et al. Enrichment of circulating and mucosal cytotoxic CD8 + Tcells is associated with postoperative endoscopic recurrence in patients with crohn's disease. J. Crohns Colitis 10, 338-345 (2016).

27. McDermott, A.J. \& Huffnagle, G.B. The microbiome and regulation of mucosal immunity. Immunology 142, 24-31 (2014).

28. Jalanka-Tuovinen, J. et al. Intestinal microbiota in healthy adults: temporal analysis reveals individual and common core and relation to intestinal symptoms. PLoS One 6, e23035 (2011).

29. Collins, S.M. A role for the gut microbiota in IBS. Nat. Rev. Gastroenterol. Hepatol. 11, 497-505 (2014).
30. Borovkova, N. et al. Influence of sexual intercourse on genital tract microbiota in infertile couples. Anaerobe 17, 414-418 (2011).

31. Hou, D. et al. Microbiota of the seminal fluid from healthy and infertile men. Fertil. Steril. 100, 1261-1269 (2013).

32. Noguera-Julian, M. et al. Gut microbiota linked to sexual preference and HIV infection. EBioMedicine 5, 135-146 (2016).

33. Lozupone, C.A. et al. HIV-induced alteration in gut microbiota: driving factors, consequences, and effects of antiretroviral therapy. Gut Microbes 5, 562-570 (2014).

34. Alam, A. etal. The microenvironment of injured murine gut elicits a local prorestitutive microbiota. Nat. Microbiol. 1, 1-7 (2016).

35. Oh, J.Z. et al. TLR5-mediated sensing of gut microbiota is necessary for antibody responses to seasonal influenza vaccination. Immunity 41, 478-492 (2014).

36. Gallo, M.F. et al. Biological markers of sexual activity: tools for improving measurement in HIV/sexually transmitted infection prevention research. Sex. Transm. Dis. 40, 447-452 (2013).

37. Rice, C.E. et al. Prostate-specific antigen is unlikely to be a suitable biomarker of semen exposure from recent unprotected receptive anal intercourse in men who have sex with men. Sex. Transm. Dis. 41, 377-379 (2014).

38. Heath, J.P. Epithelial cell migration in the intestine. Cell Biol. Int. 20, 139-146 (1996).

39. van der Flier, L.G. \& Clevers, H. Stem cells, self-renewal, and differentiation in the intestinal epithelium. Annu. Rev. Physiol. 71, 241-260 (2009).

40. Mylvaganam, G.H. et al. Diminished viral control during simian immunodeficiency virus infection is associated with aberrant PD-1hi CD4 T cell enrichment in the lymphoid follicles of the rectal mucosa. J. Immunol. 193, 4527-4536 (2014).

41. Marioni, J.C., Mason, C.E., Mane, S.M., Stephens, M. \& Gilad, Y. RNAseq: an assessment of technical reproducibility and comparison with gene expression arrays. Genome Res. 18, 1509-1517 (2008).

42. Dobin, A. et al. STAR: ultrafast universal RNA-seq aligner. Bioinformatics 29, 15-21 (2013).

43. Halpin, A.L. et al. Intestinal microbiome disruption in patients in a long-term acute care hospital: a case for development of microbiome disruption indices to improve infection prevention. Am. J. Infect. Control 44, 830-836 (2016).

44. Zhang, J., Kobert, K., Flouri, T. \& Stamatakis, A. PEAR: a fast and accurate Illumina Paired-End reAd mergeR. Bioinformatics 30, 614-620 (2014).

45. Caporaso, J.G. et al. QllME allows analysis of high-throughput community sequencing data. Nat. Methods 7, 335-336 (2010).

46. Rideout, J.R. et al. Subsampled open-reference clustering creates consistent, comprehensive OTU definitions and scales to billions of sequences. PeerJ 2, e545 (2014).

47. Kopylova, E., Noe, L. \& Touzet, H. SortMeRNA: fast and accurate filtering of ribosomal RNAs in metatranscriptomic data. Bioinformatics 28, 3211-3217 (2012).

48. Caporaso, J.G. et al. PyNAST: a flexible tool for aligning sequences to a template alignment. Bioinformatics 26, 266-267 (2010).

49. Price, M.N., Dehal, P.S. \& Arkin, A.P. FastTree: computing large minimum evolution trees with profiles instead of a distance matrix. Mol. Biol. Evol. 26, 1641-1650 (2009).

50. DeSantis, T.Z. et al. Greengenes, a chimera-checked 16S rRNA gene database and workbench compatible with ARB. Appl. Environ. Microbiol. 72, 5069-5072 (2006).

51. Langille, M.G. et al. Predictive functional profiling of microbial communities using 16S rRNA marker gene sequences. Nat. Biotechnol. 31, 814-821 (2013).

52. Parks, D.H. \& Beiko, R.G. Identifying biologically relevant differences between metagenomic communities. Bioinformatics 26, 715-721 (2010).

53. Golden, M.R. et al. Herpes simplex virus type 2 (HSV-2) Western blot confirmatory testing among men testing positive for HSV-2 using the focus enzyme-linked immunosorbent assay in a sexually transmitted disease clinic. Sex. Transm. Dis. 32, 771-777 (2005).

54. Lozupone, C. \& Knight, R. UniFrac: a new phylogenetic method for comparing microbial communities. Appl. Environ. Microbiol. 71, 8228-8235 (2005).

55. Clarke, K.R. Non-parametric multivariate analyses of changes in community structure. Aust. J. Ecol. 18, 117-143 (1993). 